courbe (2), et réciproquement. Quand la courbe des débits est de la forme

$$
Q=\frac{A}{(1+x t)^{2}} \quad(\text { A débit au temps } t=0),
$$

les courbes ( 1 ) sont de la forme

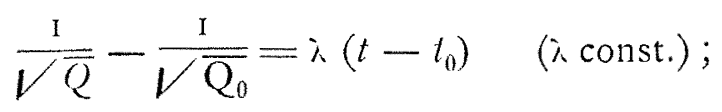

elles sont convexes et passent par l'origine. La réciproque est vraie. Quand la courbe des débits est de la forme

$$
Q=A e^{-\alpha t}, \quad(x>0),
$$

les courbes (1) sont des droites passant par l'origine,

$$
Q=Q_{0} e^{-\alpha\left(t-t_{1}\right)}
$$

"La réciproque est vraie $\left({ }^{\star}\right)$.

( Ces formules (3) et (5) sont d'ailleurs exactes en théorie (Boussinese, Comptes-rendus, juin et juillet r go3). M. Boussinesq a encore indiqué la loi (Comptes-rendus, 20 juillet 1903)

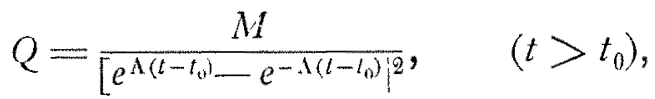

qui se réduit sensiblement à la loi (6) si $A$ ou $t-t_{0}$ est assez grand.

"Enfin si, pour une source, on peut déterminer une suite de périodes où la nappe ne reçoit pas d'apports extérieurs et où il existe une courbe des débits, le débit $Q$ étant fonction décroissante du temps, l'équation $d V=-Q d t$ et l'équation (2) montrent que $V$ est fonction croissante de $Q$.

"Noủs indiquerons, dans une autre communication, des applications et des vérifications expérimentales des considérations précédentes.

(1) Dans un Mémoire manuscrit présenté par nous à l'Académıe des Sciences à la fin de mai 1903 et retiré depuis, nous avions indiqué les équations ( 1 ) et (2) et un certain nombre de leurs propriétés annsi que la notion de courbe des débits. Grâce à des calculs basés sur des hypothèses qui, ainsi que nous l'a fait remarquer $M$. Boussinesq, sont critiquables, nous obtenıons : ${ }^{\circ}$ pour les nappes à fond horizontal dans la période de régime non influencé la formule $Q=\frac{A}{(1+x t)^{2}}$ établie par M. Boussinesq dans sa Communication du 6 juillet Igo3, la formule $Q=\lambda_{1} \frac{h^{2}}{L}\left(\lambda_{1}\right.$ const.) analogue à la formule $(14) \mathrm{de}$ la même Communication, mais avec une valeur moins exacte des constantes $)_{1}$ et $A$; enfin la formule (4) ci-dessus; $2^{\circ}$ pour les nappes à fond rectiligne incliné, la formule $Q=A C-\alpha t$ analogue à une formule établie par $M$. Boussinesq dans sa Communication du 22 juın I 903 (aux constantes près) ; enfin la formule (6) ci-dessus. Pratiquement, tant que les valeurs des constantes sont déterminées à l'aide des débits fournis par l'expértence, nos formules ci-dessus suffisent. Elles ont d'alleurs eu un rôle utıle, car ce sont elles qui nous ont conduit aux graphiques de prévision des débits des sources de Cérilly et Armentières (Vanne) dont il sera question dans une Communication ultérieure.

Le prochain numéro contiendra un article concernant les essais de traction électrique sur la ligne de Saint-Georges-deComiers à La Mure (Isère) avec la locomotive "Le Drac", par M. Barbillion, sous-directeur de l'Institut électrotechnique de l'Université de Grenoble.

\section{Deux nouveaux débouchés de la Houille blanche}

\section{I. - FABRIGATION DES GYANAMIDES ET LEUR EMPLOI EN AGRIGULTURE.}

Un regain de vie, un renouveau de prospérité seraient-ils sur le point de donner à l'industrie du carbure de calcium une nouvelle actıvité et même d'étendre, au-delà de toute espérance, l'importance économıque de cette branche de l'Electrochimie? Il serait peut-être prématuré de l'affirmer d'une manière abso. lue, ma1s quelques-unes des conférences faites au $\mathrm{V}^{\circ}$ Congrès de Chimie appliquée à Berlin, ont éveillé, à juste titre, l'atten tion des fabricants de carbure et leur permettent l'espoir très prochainement réalisable, semble-t-1l - d'un développe. ment qu'ils attendent depuis plusieurs années.

Un des artıcles récents de cette Revue (I) signalait commeni les Américains savent tirer des carbures, et notamment dı carbure de baryum, les cyanures alcalino-terreux et alcalins. En falsant passer de l'azote, préparé à partir de l'air, sur un carbure probablement rendu alcalin, solt par un excès d'oxyde, soit par adjonction d'un alcali, à une température élevée, on obtient leur union et l'on retire du four un cyanure alcalinoterreux - de baryum - qu'on transforme ensurte, par double décomposition, en cyanure de potassium qui est la forme d'utilisation industrielle des cyanures.

Mais ce n'est point de cette opération qu'll est question dans le cas présent. La consommation du cyanure de potassium en nature tend plutôt à diminuer qu'à augmenter ; la transformation des cyanures en ammoniaque n'est qu'à l'étude, et leur oxydation les transformant en cyanates n'est sans doute pas aussi facilement réalisable qu'on pourratt en juger d'après certains brevets ; d'ailleurs, la valeur de ces cyanates comme engrais azotés, ne paraît pas avorr été démontrée par des essais suffisants. Le sens des réactions présentées au Congrès de Berlin par M. ERLWEIN, d'après les études de MM. Franck, CARO et ROTHE, est néanmoins biell voisin du précédent.

Si l'on fart passer de l'azote sur du carbure de calcium pulvérisé, porté à une température assez élevée, on arrive non pas à un cyanure, mais au sel de calcium de la Cyanamide, répondant à la formule $\mathrm{CaCAz}^{2}$. Ces savants, poursuivant leurs études avec l'appui de la maison Siemens, ont, d'ailleurs, réussi à préparer ce prodult directement, dans un four électrique à résistance, à partır de la chaux, du charbon et de l'azote, sans isoler le carbure de calcium, corps intermédiaire, de sorte que cette préparation est ainsi considérablement simplifiée. Or, ce CYANAMIDE-CALCIUM, sous l'influence de la vapeur d'eau sous pression, donne de l'ammoniaque et du carbonate de chaux ; par lixiviation à l'eau il se transforme en chaux et en drcyandmide, substance de formule $\mathrm{C}^{2} \mathrm{Az}^{4} \mathrm{H}^{4}$, qui est susceptible par une fusion avec le carbonate de soude, de donner de l'ammoniaque d'une part et, d'autre part, du cyanure de sodium. Il est vrai qu'alors une partie assez notable (un quart) de l'azote, fixé d'abord, est perdu sous la forme élémentarre et qu'une autre partie, mais plis fable, passe à l'état de mélamine ou de tricyanamade, qu'il faut traiter ultérieurement pour en tirer des produtts utilisables. Enfin, une fusion du même corps avec du sel marın donne également un cyanure assez riche pour recevoir des utilisations immédiates.

Mais en outre, et surtout, le Cyanamide-calcium brut peut être utılisé directement à la fumure des terres, comme véhicule de l'azote ammoniacal et à un prix qui lui permet avantageusement la lutte avec les autres formes d'emploi de cet élément. MM. GERLACH et WAGNER ont étudié en détail l'action de cet " azote-chaux " sur la végétation : à teneur d'azote égale, son effet sur tous les genres de culture est à peu près équivalent à l'azote ammoniacal et à peine inférieur à l'azote nitrique. Il est probable qu'il est d'abord transformé

(I) « L'Electrochimie sur les bords du Niagara », no 8 , page 235 . 
en ammoniaque dans le sol, puis nitrifié, et c'est ainsi qu'il agirait sur l'économie de la plante. Il présente une teneur moyenne en azote, comparable à celle du salpêtre ou du sulfate d'ammoniaque. L'effet de ses deux dérivés, la cyanamide et lo dicyanamide, beaucoup plus riches en azote, n'a pas été aussi bien détermıné ; mais, suivant toute vraisemblance, ils agisseñ eux aussi d'une manière analogue.

Ainsi, voilà donc l'azote atmosphérique fixé directement au four électrique d'une manière industrielle et livré à l'agriculture sous une des formes qu'elle apprécie le plus. Est-ce là le produit de consommation illimité dont parlait un des rédacteurs de cette Revue, et dont l'obtention doit occuper, enfin, tant de chevaux hydrauliques disponibles dans nos torrents ? Constatons une fois de plus que ce sont les Allemands - quı n'ont pas de chutes d'eau — qui poussent le plus activement l'étude de ces questions, tandis que chez nous - où l'on ne sait que farre de nos forces hydrauliques - on semble s'en désıntéresser | Voicı que, grâce à l'air liquide que les procédés Claude, à l'heure actuelle industriellement au point, donnent à rasson de plus de I kilog. par cheval-heure, on peut, d'une part, obtenır l'azote pour les cyanamides et, d'autre part, l'oxygène pour les nitrates, à des prix d'extrême bon marché C'est une avance énorme que nous avons sur nos vorsins. Ne va-t-on pas enfin en profiter? Notre industrie ne se déc1dera-t-elle à aborder ces nouvelles branches que lorsque les meilleures places seront prises ?.

\section{II. - LE TÉTRAGHLORURE DE GARBONE,}

\section{SA FABRICATION ET SES EMPLOIS INDUSTRIELS.}

La principale cause qui limite le développement de l'ındustrie de la soude électrolytique réside dans la difficulté qu'éprouvent les usines productrices à se défarre de leur chlore Car, annsi que le fait observer le docteur Lunge : "Même dans la grande majorité des cas, lorsqu'on a effectivement pour objet la production de la soude caustique ou celle du carbonate, ces derniers produits, quorque constituant des facteurs très importants pour l'économie du procédé, ne tiennent certainement que le second rang, non seulement au point de vue financier, mais aussi sous le rapport quantitatıf. La fabrication du chlore par l'électrolyse ne peut, dans aucun cas, être envisagée comme une branche accessoire de la fabrication de la soude, comme elle l'était généralement autrefois dans le procédé Leblanc, et comme elle peut l'être encore aujourd'hui, dans une certaine mesure; c'est précisément l'inverse qui a lieu $n$ (I). On trouve, d'autre part, la confirmation de ce fait dans la conférence de M. Gall au Congrès de la Houlle Blanche, sur "L'état actuel de l'Industrie électrochimıque " $\mathrm{Si}$ l'on remarque, a dit le conférencier, que le principal emploi pratique connu du chlore est de se combiner à la chaux pour former un hypochlorite, on volt qu'à la production d'une tonne de soude correspond la fabrication de plus de deux tonnes d'hypochlorite de chaux. Or, l'emploi de la soude est, pour ansi dire, illimité; 11 s'en consomme en France, environ 180.000 tonnes par an; tandis que l'hypochlorite de chaux qui n'est guère employé que pour le blanchîment de la pâte à papier et la désinfection des locaux insalubres a un emploi très limité ; 11 ne s'en consomme pas même 20.000 tonnes en France. Dans ces conditions, une usine électrolytique qui produirait 8 à 10.000 tonnes de soude, trouverait à peine, à elle seule, à écouler tout son chlore.

Ces usines ne peuvent donc guère vivre en se confinant dans la préparation de l'alcal 1 ; on en cite même qui, au contraire, se bornent à rechercher les débouchés du chlore et laissent perdre leurs lessives D'ailleurs, voudrait-on rejeter le chlore qu'on serait obligé dans ce but, d'établir des appareils compliqués

(1) "Fabrication Electrolytique de la Soude, du Chlore, des Liqueurs de blanchiment et des Chlorates ", par le Dr Lunge, professeur à l'Ecole Polytechnique de Zurich. et coûteux et exigeant une survellance incessante. Aussi, pour développer leur fabrication, les usines de soude électrolytique ont-elles cherché à faıre revêtur au chlore des formes s'appliquant à d'autres usages qu'au blanchîment et à la désinfection.

Parmi ces formes est celle du tétrachlorure de carbone $\mathrm{CCl}^{k}$, produit très avantageux à beaucoup d'égards. D'abord, c'est un corps quı est, de par sa constitution même, formé presque entièrement de chlore, 1 en renferme exactement 92,20 pour Ioo. Liquide incolore, d'odeur éthérée, de densité 1,72 , 1 est d'une manipulation incomparablement moins désagréable quc le chlore et n'est pas toxique comme ce gaz dont 1 l ne rappelle en rien l'odeur asphyxiante; enfin, il ne nécessite pas, pour son expédition, le transport d'un pords mort considérable comme celu de la chaux dans l'hypochlorite de chaux.

Ce nouveau produit est susceptible de prendre une assez grande importance économique en tant que dissolvant des corps gras; facilement volatıl, on commence à l'employer pour remplacer la benzine, le sulfure de carbone et les éthers de pé. trole dans certaines fabriques que ces derniers corps 11ennent dans une menace continuelle d'incendre. Le tétrachlorure de carbone, en effet, est parfaitement incombustible et ses vapeurs ne forment avec l'aur aucun de ces mélanges explosıfs auxquels exposent les produits précités. Il semble donc avoir un large débouché dans les nouvelles usınes qui pratıquent le décraissage à sec en vase clos et surtout dans les fabriques d'hule où 1l se substitue avantageusement au sulfure de carbone pour l'épursement des tourteaux. D'autres applications sont en vore d'essai qui étendront sans doute encore son emplor et développeront sa fabrication industrielle.

Il est toujours préparé à partir du sulfure de carbone par l'action directe ou détournée du chlore L'action directe n'a heu qu'à température élevée, ou à la température ordinarre en présence de corps agissant comme corps de contact. Dans l'action indirecte, on engage d'abord le chlore en une combinasson telle que le chlorure de soufre, par exemple, que l'on fait agir ensuite sur le sulfure de carbone en présence également de corps de contact.

Dans le premier cas, le chlore se combine d'une part, au soufre du sulfure de carbone en donnant du chlorure de soufre et, d'autre part, au carbone en donnant nassance aut tétrachlorure de carbone; la réaction est exprimée par la formule

$$
\mathrm{CS}^{2}+6 \mathrm{Cl}=\mathrm{S}^{2} \mathrm{Cl}^{2}+\mathrm{CCl}^{*}
$$

Les points d'ébullition du chlorure de soufre et du tétrachlorure de carbone étant assez élorgnés, on sépare facilement à quelques centièmes près, ces liquides par distıllation et rectification; on purifie ensuite le tétrachlorure par des lavages alcalins On a intérêt à ne plus avoir de sulfure de carbone au moment de la rectification, mais dans le cas oì il s'en trouverat encore, il suffit pour le détruire entièrement, d'insuffler du chlore dans le liquide en présence d'un corps de contact convenable, la transformation dans ce cas élant quantilative.

Dans la méthode indrecte : action du chlorure de soufre sur le sulfure de carbone en présence d'un corps de contact, le chlore du chlorure s'unit au carbone du sulfure duquel le soufre libéré se dissout dans le tétrachlorure au fur et à mesure de sa formation. Cet échange est représenté par la formule :

$$
\mathrm{CS}^{2}+2 \mathrm{~S}^{2} \mathrm{Cl}^{2}=\mathrm{CCl}^{4}+6 \mathrm{~S} \text {. }
$$

Le soufre cristallise à froid ct l'on recuellle le tétrachlorure par simple décantation. Il est séparé du chlorure de soufre ct du sulfure de carbone entrainés comme 1] vient d'ĉtre dit.

Un premier procédé de fabrication consiste à faire circuler un mélange de vapeurs de sulfure de carbone et de chlore daras des tubes portés à la température du rouge vif (I). La plus grande partie du mélange est transformée en chlorure de soufre et tétrachlorure de carbone, mais la réaction est limitée par

(1) Kolbe. Ann. d. Chemie 4 . Pharm. 15, 41, et id. 51, 147. 
la décomposition, à cette température, du tétrachlorure en hexachloroéthane, tétrachloroéthène, et par la formation de produits chlorosulfurés desquels on peut cependant retirer encore du tétrachlorure (I).

Ces réactions secondarres et l'obligation d'opérer à haute température ont fait, jusqu'à ces dernières années, préférer, dans la pratique, les procédés par l'influence des substances de contact.

En faisant barboter, à la température ordinaire, le chlore dans du sulfure de carbone contenant de l'iode, du pentachlorure d'antımoine ou du chlorure de molybdène (2), la même transformation peut s'opérer sans qu'on ait l'inconvénient de la destruction d'une partie du tétrachlorure formé; mais 11 reste, avec le tétrachlorure, des composés chlorosulfurés qu'il faut détruire ensuite; puis dans la séparation du chlorure de soufre et du tétrachlorure, on perd la substance active qu'il faut remplacer à chaque opération Nombreux sont les dispositıfs plus ou moins pratıques imagınés pour réaliser industriellement cette opération.

Un moyen indirect d'arriver à ce même résultat échappe à ce dernier désavantage, mass toutefors sans supprimer complètement le premier Le procédé consıste à former d'abord du chlorure de soufre par unıon directe du chlore et du soufre à sa température de fusion et ensuite à mélanger à ce chlorure de soufre, à la température de $80^{\circ}$ ou $100^{\circ}$ et en présence de limalle de fer, du sulfure de carbone (3), de manière que ce dernier soit en défaut. Il se transforme alors en tétrachlorure de carbone et soufre comme 1 l a été expliqué plus haut; les produits chlorosulfurés paraissent être moins abondants dans ce genre d'opération

Plus récemment, on a tenté, avec succès, de combiner cette dernière réaction avec l'une des premières pour élıminer le chlorure de soufre qui prend narssance dans cclles-ci et n'avoir finalement que du tétrachlorure de carbone. En même temps, on a imaginé des appareils permettant de rendre partiellement $(t)$ ou cotalement (5) continue la marche de ces opératıons. On a obtenu ces résultats en employant des substances de contact différentes de celles précédemment citées et l'on a choisi soit le chlorure d'aluminium pulvérısé (4), soit mieux encore, du coke imprégné de chlorure manganeux sec (5) qui ne peuvent pas gêner ou même ne sont pas entraînés par les masses réagissantes C'est dans cette dernière voie que les mellleurs résultats pratiques ont été obtenus jusqu'ici. On fait tomber le sulfure de carbone sur des fragments de colke imprégnés de chlorure manganeux remplissant une colonne verticale au bas de laquelle arrive le chlore; la réaction se produit ainsı méthodiquement d'une manière continue.

Le mélange de chlorure de soufre et de tétrachlorure de carbone, formé par l'action directe du chlore sur le sulfure de carbone est alors, suivant les procédés, tantôt séparé par distillation pour soumettre le chlorure de soufre à l'action du sulfure de carbone en présence de la limaille de fer (brevet allemand 72999) dans un récipient chauffé; tantôt admis, au fur et à mesure de sa formation, sur des fragments de coke imprégnés d'un sulfure métallique emplissant une colonne verticale,maintenue à $80^{\circ} \mathrm{C}$, au bas de laquelle on injecte du sulfure de carbone qui, se vaporisant aussitôt, réagit méthodiquement et d'une manière continue sur le chlorure de soufre (brevet français $316.97 \mathrm{I}$ ) Ce dernier brevet indique la séparation des produits, issus de cette colonne, par chauffage dans une cornue à $150^{\circ}$ : le tétrachlorure de carbone volatisé est recueillı par condensation dans un serpentin, le chlorure de soufre entraîné

(1) L. Meyer. Berichte d. deutschen Chem. Gesell. 20, 682. Gustavson, id. 3, 989. -- Klason, id. 20, 2383.

(2) Hofrmann, Ann. d. Chemie u. Pharm. 115, 264. - K Lason, Berichte d. deutschen Chem. Gesell. 20, 2376 .

(3) Brevet allemand, no 72.999 , I 8 avril 1893.

(4) Brevet français, no 308.916, I2 mai Igor.

(5) Brevet français, no $316.97 \mathrm{x}, \mathrm{1} 3$ décembre 1902. fait retour à la partie supérieure de la colonne et le soufre libéré étant fondu coule au fond d'une cornue à sulfure de carbone et est ainsi remis automatiquement en réaction. L'appareil est, en somme, sous une forme simple et qui dort être très peu encombrante, imaginé pour qu'on n'ait à y introdure que du chlore et du charbon.

Enfin, un procédé fort ingénieux (I), mais dont les preuves pratiques ne sont pas encore faites, reprend l'action du chlore sur le sulfure de carbone au rouge, afin d'obtenir dans un appareil unique, le tétrachlorure à partir du chlore et du charbon. Son principe, facile à expérimenter en laboratorre, est d'envoyer du chlore dans une cornue chauffée au rouge qui contient du charbon et un peu de soufre. Ces deux derniers éléments donnant d'abord du sulfure de carbone, on obtient ainsi du chlorure de soufre et du tétrachlorure. En ramenant par un dispositif approprié le chlorure de soufre constamment dans la cornue, il s'y dissocie,met en liberté son chlore et son soufre qui reproduit du sulfure de carbone, de sorte que la réaction se poursuit toujours suivant ce même cycle et donne comme résul. tat final du tétrachlorure Malheureusement, le produit obtenu par cette voie est loin d'être pur et l'on y retroure non seulement les impuretés qui l'accompagnent dans les procédés par action indirecte, mais même celles auxquelles donnent natssance les méthodes par action directe du chlore sur le sulfure de carbone au rouge et qui sont dues au chauffage à haute température du tétrachlorure.

Quoiqu'1l $€$ n soit de la plus ou moins grande simplicité de ces méthodes et malgré les lavages aux alcalis ou à la chaux, les rectifications multiples en présence d'eau, sous pression, ou dans le vide auxquelles on doit soumettre le tétrachlorure de carbone afin de l'obtenir rigoureusement pur pour certaines applications, c'est un produit déjà industriel et qui se fabrique par I 5 ou 20 tonnes par mois en France. Ceci montre que la consommation en est déjà appréciable et elle ne peut aller qu'en augmentant dans de larges proportions en raison des avantages que ce produit nouveau possède sur les dissolvants industriels employés jusqu'ici. On ne peut pas sans doute en attendre une renaissance des affaires de soude électrolytique, mais, tout au moins, il favorisera beaucoup l'extension et le développement de celles qui ont subsisté jusqu'icı.

UN ABONNÉ,

Electrochimiste industriel.

\section{DE L'INTERVENTION DE L'ÉLECTROCHIMIE} DANS LA MÉTALLURGIE DU PLOMB (Ancienne méthode d'exploitation de Pontgibaud) (Suite)

\section{III. - RÉDUCTION DES MINERAIS}

L'opération de la réduction des minerais suivait immédiatement celle du grillage. Elle consistait à transformer les oxydes et les silicates de plomb formés pendant le grillage scorifiant en plomb d'œuvre et scories dont on se débarrassait. La situation et la disposition de l'atelier de la réduction du ininerai ayant été décrites dans l'article précédent nous n'y reviendrons pas pour l'instant. Nous allons décrire maintenant les appareils de cette opération, afin de renseigner aussi exactement que possible sur les réactions chimiques qui avaient licu entre les divers corps mis en présence, et sur les résultats de ces phénomènes.

Description des appareils. - A Pontgibaud, pour opérer la réduction du minerai grillé, on employait des demi-

(1) Brevet français, no $312.046,22$ juin rgot. 